\title{
Proangiogenic functions of an RGD-SLAY-containing osteopontin icosamer peptide in HUVECs and in the postischemic brain
}

\author{
Hahnbie Lee ${ }^{1,2}$, Yin-Chuan Jin ${ }^{1,3}$, Seung-Woo Kim ${ }^{1,2}$, Il-Doo Kim ${ }^{1,2}$, Hye-Kyung Lee ${ }^{1,2}$ and Ja-Kyeong Lee Le, $^{1,2}$ \\ Osteopontin (OPN) is a phosphorylated glycoprotein secreted into body fluids by various cell types. OPN contains arginine-glycine- \\ aspartate (RGD) and serine-leucine-alanine-tyrosine (SLAY) motifs that bind to several integrins and mediate a wide range of cellular \\ processes. In the present study, the proangiogenic effects of a 20-amino-acid OPN peptide (OPNpt20) containing RGD and SLAY \\ motifs were examined in human umbilical vein endothelial cells (HUVECs) and in a rat focal cerebral ischemia model. OPNpt20 \\ exerted robust proangiogenic effects in HUVECs by promoting proliferation, migration and tube formation. These effects were \\ significantly reduced in OPNpt20-RAA (RGD- > RAA)-treated cells, but only slightly reduced in OPNpt20-SLAA (SLAY-> SLAA)- \\ treated cells. Interestingly, a mutant peptide without both motifs failed to induce these proangiogenic processes, indicating that the \\ RGD motif is crucial and that SLAY also has a role. In OPNpt20-treated HUVEC cultures, AKT and ERK signaling pathways were \\ activated, but activation of these pathways and tube formation were suppressed by anti- $\alpha_{v} \beta_{3}$ antibody, indicating that OPNpt20 \\ stimulates angiogenesis via the $\alpha_{v} \beta_{3}$-integrin/AKT and ERK pathways. The proangiogenic function of OPNpt20 was further confirmed \\ in a rat middle cerebral artery occlusion model. Total vessel length and vessel densities were markedly greater in OPNpt20-treated \\ ischemic brains, accompanied by induction of proangiogenic markers. Together, these results demonstrate that the 20-amino-acid \\ OPN peptide containing RGD and SLAY motifs exerts proangiogenic effects, wherein both motifs have important roles, and these \\ effects appear to contribute to the neuroprotective effects of this peptide in the postischemic brain. \\ Experimental \& Molecular Medicine (2018) 50, e430; doi:10.1038/emm.2017.241; published online 19 January 2018
}

\section{INTRODUCTION}

Osteopontin (OPN) is a secretory phosphoprotein expressed in various tissues, including the brain, and it has been reported that OPN has important roles in various physiological processes, such as anchoring osteoclasts to the mineral bone matrix during bone resorption ${ }^{1}$ and attaching calcium oxalate crystals to renal tubules in stone formation. ${ }^{2}$ Elevation of OPN expression has been observed in various pathological conditions, including atherosclerosis, ${ }^{3}$ multiple sclerosis, ${ }^{4}$ rheumatoid arthritis ${ }^{5}$ and most cancers, ${ }^{6}$ which suggests that OPN has an important role in these conditions. In the normal brain, OPN expression is weak, but under pathological conditions, it is markedly upregulated in microglia and astrocytes. ${ }^{7}$ In particular, delayed but significant induction of OPN has been reported in ischemic stroke; for example, in a rat model of transient forebrain ischemia and in a mouse model of permanent focal ischemia, OPN induction began at $12 \mathrm{~h}$ after injury and peaked at 5 days, respectively. ${ }^{8,9}$
OPN contains a highly conserved arginine-glycine-aspartic acid (RGD) motif in its $\mathrm{N}$-terminal region, which interacts with $\alpha_{\mathrm{v}} \beta_{1^{-}}, \quad \alpha_{\mathrm{v}} \beta_{3^{-}}$and $\alpha_{\mathrm{v}} \beta_{5^{-}}$-integrin. ${ }^{10}$ In addition, the SVVYGLR motif, which is adjacent to the RDG motif, is exposed by thrombin cleavage and binds to $\alpha_{9} \beta_{1^{-}}, \alpha_{4} \beta_{1^{-}}, \alpha_{4} \beta_{7^{-}}$ and $\alpha_{v} \beta_{3}$-integrin. ${ }^{11,12}$ These OPN-integrin interactions mediate cell-cell and cell-matrix interactions and modulates a wide range of cellular processes, including adhesion, migration and survival, ${ }^{13}$ and have therefore been implicated in various diseases. In addition to integrins, OPN also binds to CD44 hyaluronate receptor, a transmembrane glycoprotein known to mediate inflammatory processes, cell adhesion, and cell migration $^{14}$ via its CD44-binding domain located in the C-terminal region. ${ }^{15}$

In our previous study, we reported that an intranasally delivered RGD and serine-leucine-alanine-tyrosine (SLAY) motif-containing OPN icosamer peptide (20-amino-acid OPN peptide (OPNpt20)) exerted a robust neuroprotective

\footnotetext{
${ }^{1}$ Department of Anatomy, Inha University School of Medicine, Inchon, Republic of Korea; ${ }^{2}$ Medical Research Center, Inha University School of Medicine, Inchon, Republic of Korea and ${ }^{3}$ Department of Histology and Embryology, Binzhou Medical University, Yantai, China Correspondence: Professor J-K Lee, Department of Anatomy, Inha University School of Medicine, Inharo 100 Nam-gu, Inchon 22212, Republic of Korea. E-mail: jklee@inha.ac.kr

Received 8 December 2016; revised 5 July 2017; accepted 7 July 2017
} 
a

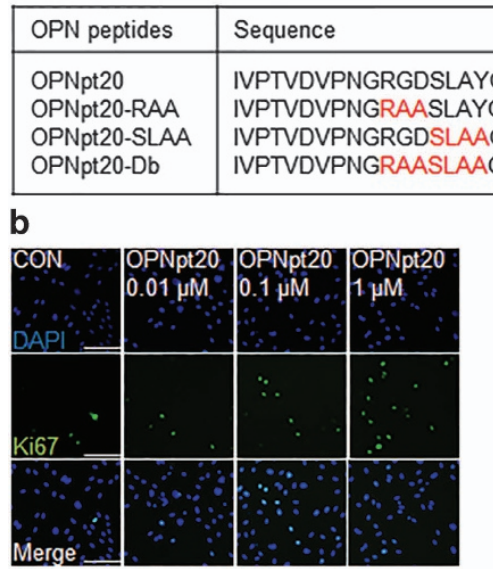

e

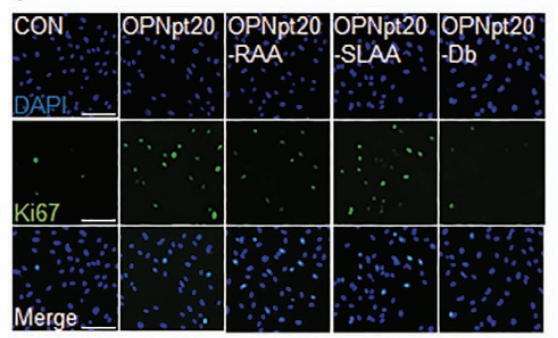

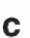

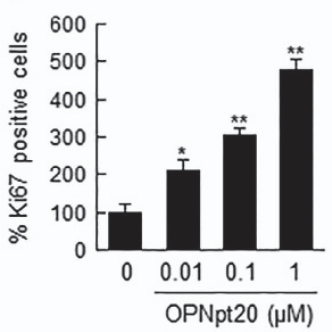

f

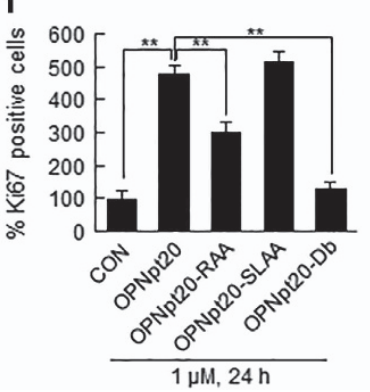

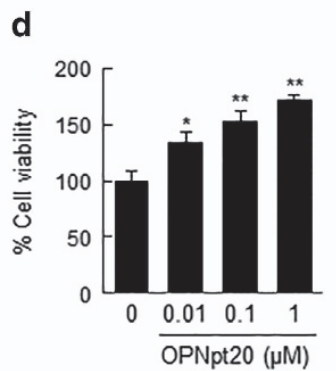

g

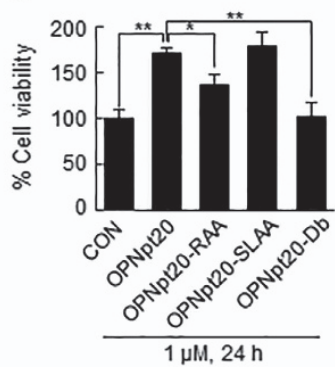

Figure 1 Induction of cell proliferation by 20-amino-acid OPN peptide (OPNpt20) and its mutants in human umbilical vein endothelial cell (HUVEC) cultures. (a) Amino-acid sequences of OPNpt20 and its three mutants. (b, c) Cell proliferation was visualized by immunofluorescent staining using anti-Ki67 antibody in HUVECs treated with OPNpt20 (0.01, 0.1 or $1 \mu \mathrm{m})$ for $24 \mathrm{~h}$. Ki67 indices were determined by counting numbers of Ki67-positive cells among DAPI (4',6-diamidino-2-phenylindole)-positive cells in five high-power fields per plate in three plates. (e, f) HUVEC proliferation was visualized by immunostaining with anti-Ki67 antibody after treating HUVECs for $24 \mathrm{~h}$ with either of the three mutant peptides $(1 \mu \mathrm{m})$. Representative images are presented $(\mathbf{b}, \mathbf{e})$ and results are presented as the mean \pm s.e.m. $(n=3)$ (c, f). (d, g) MTT (3-(4,5-dimethylthiazol-2-yl)-2,5-diphenyl tetrazolium bromide) assays were conducted under the same conditions used in (b, e), and results are presented as the mean \pm s.e.m. $(n=4)$. Scale bars, $200 \mu \mathrm{m}$. ${ }^{*} P<0.05$, ${ }^{* *} P<0.01$ between the indicated groups.

effect in the postischemic brain because of its antiinflammatory effects. ${ }^{16}$ In this previous study, OPNpt20 was found to significantly suppress inducible nitric oxide synthase (iNOS) induction and nitrite production in a rat model of focal cerebral ischemia (middle cerebral artery occlusion, MCAO) and in lipopolysaccharide-treated primary microglial cultures, and the results obtained suggested that the interaction between the RGD motif of OPNpt20 and $\alpha_{v} \beta_{3}$-integrin has a critical role. In the present study, we investigated the proangiogenic potential of OPNpt20 in human umbilical vein endothelial cells (HUVECs) and in a rat MCAO model. In addition, we explored the molecular mechanism behind the proangiogenic effects of OPNpt20 using three mutant peptides (RGD $\rightarrow$ RAA, SLAY $\rightarrow$ SLAA and RGDSLAY $\rightarrow$ RAASLAA) and an $\alpha_{\mathrm{v}} \beta_{3}$-integrin-blocking antibody.

\section{MATERIALS AND METHODS}

\section{Surgical procedure used for MCAO}

Male Sprague-Dawley rats were housed under diurnal lighting conditions and allowed food and tap water ad libitum. All animal studies were carried out in strict accordance with the recommendations made in the Guide for the Care and Use of Laboratory Animals published by the National Institute of Health (2013) and ARRIVE guidelines (http://www.nc3rs.org/ARRIVE). The animal protocol used was reviewed and approved by the INHA University-Institutional Animal Care and Use Committee (INHA-IACUC; Approval Number INHA-160824-434). MCAO was carried out as described previously. ${ }^{17}$ In brief, 8-week-old male Sprague-Dawley rats $(250-300 \mathrm{~g})$ were anesthetized with $5 \%$ isoflurane in $30 \%$ oxygen $/ 70 \%$ nitrous oxide and maintained using $0.5 \%$ isoflurane in the same gas mixture during surgery. Occlusion of the right middle carotid artery was induced for $1 \mathrm{~h}$ by advancing a nylon suture (4-0; AILEE, Busan, Korea) with a heat-induced bulb at its tip ( $\sim 0.3 \mathrm{~mm}$ in diameter) along the internal carotid artery for 20-22 mm from its bifurcation with the external carotid artery. This was then followed by reperfusion for up to 7 days. A thermoregulated heating pad and a heating lamp were used to maintain a rectal temperature of $37 \pm 0.5^{\circ} \mathrm{C}$ during surgery. Animals were randomly allocated to sham, $\mathrm{MCAO}+$ phosphate-buffered saline (PBS), MCAO+OPNpt20 or MCAO+OPNpt20-Db (mutant OPN peptide with both RGD and SLAY replaced) groups. Animals allocated to the sham group underwent an identical procedure, but the MCA was not occluded. 


\section{Peptide treatment}

OPNpt20 and three mutant peptides (Figure 1a) were customsynthesized by Peptron (Daejeon, South Korea). Rats were anesthetized with an intramuscular injection of a ketamine $(3.75 \mathrm{mg} / 100 \mathrm{~g}$ body weight) and xylazine hydrochloride $(0.5 \mathrm{mg} / 100 \mathrm{~g}$ body weight $)$ mixture. Peptides $(1.7 \mu \mathrm{g} / 100 \mathrm{~g}$ body weight) were dissolved in $30 \mu \mathrm{l}$ of PBS $(0.01 \mathrm{M})$ and injected intranasally at 4, 5 and 6 days after MCAO. Brain tissues, cerebrospinal fluid and blood samples were collected at 7 days after MCAO.

\section{HUVEC cultures}

HUVECs were purchased from the American Type Culture Collection (ATCC, Manassas, VA, USA). Cells were grown on $100 \times 20 \mathrm{~mm}^{2}$ Petri dishes coated with $0.1 \%$ gelatin for subculture. Cultures were maintained with endothelial cell medium (Sciencell, Carlsbad, CA, USA) containing $5 \%$ fetal bovine serum (FBS), 1\% penicillin/ streptomycin and $1 \%$ endothelial cell growth supplement. Before peptide treatment, HUVEC cultures were starved in medium 199 (M199; Welgene, Gyeongsan, Korea) containing 20\% endothelial cell medium for $12 \mathrm{~h}$. Experiments were conducted using cells passaged three to eight times.

\section{MTT cell proliferation assay}

HUVEC proliferation and viability were analyzed using an MTT (3-(4,5-dimethylthiazol-2-yl)-2,5-diphenyl tetrazolium bromide) assay. Briefly, HUVEC cultures were treated with peptide for $24 \mathrm{~h}$; then, $10 \mu \mathrm{l}$ of an MTT stock solution $\left(5 \mathrm{mg} \mathrm{ml}^{-1}\right.$ in $0.01 \mathrm{M}$ PBS) was added and incubated for $1 \mathrm{~h}$ at $37^{\circ} \mathrm{C}$. Media were removed, and dimethyl sulfoxide $(200 \mu \mathrm{l} / 24$-well $)$ was added to solubilize the formazan product. After $30 \mathrm{~min}$ at room temperature, the absorbance was measured at $460 \mathrm{~nm}$.

\section{Immunocytochemistry}

A total of $1.0 \times 10^{5}$ cells per well were seeded on gelatin-coated cover glasses in 24-well plates. After starvation for $12 \mathrm{~h}$, cultures were treated with peptide in M199 medium containing 1\% FBS (GE Healthcare Life Sciences, Marlborough, MA, USA) for $24 \mathrm{~h}$ and then fixed with $4 \%$ paraformaldehyde. After washing with $0.01 \mathrm{M}$ PBS, cells were incubated in blocking solution $(0.1 \%$ Triton X-100, $2 \%$ bovine serum albumin, 5\% FBS, 5\% Normal goat serum in $0.01 \mathrm{M} \mathrm{PBS}$ ) at room temperature for $1 \mathrm{~h}$, immunostained with anti-Ki67 antibody (Abcam, Cambridge, UK) and mounted using a mounting medium with DAPI (4',6-diamidino-2-phenylindole; Vector Laboratories, Burlingame, CA, USA).

\section{Wound healing assay}

HUVECs were seeded at $2.0 \times 10^{5} /$ well onto gelatin-coated 12 -well plates and starved for $12 \mathrm{~h}$ after reaching $90 \%$ confluency. Wells were then scratched longitudinally and horizontally with a yellow tip. After washing two times with M199, cultures were treated with the same medium containing $1 \%$ FBS with or without OPNpt20. Cell migration was assayed $12 \mathrm{~h}$ after M199 treatment using a real-time cell history recorder (JuLi Stage; NanoEnTek, Seoul, South Korea). Wound widths were measured by using the ImageJ software MRI Wound Healing Tool (National Institute of Health (NIH), Bethesda, MD, USA), and percent cell motility was calculated using the following equation: $(($ area at $0 \mathrm{~h}-$ area at $12 \mathrm{~h}) /$ area at $0 \mathrm{~h}) \times 100$.

\section{Tube formation assay}

HUVECs $\left(5.0 \times 10^{4}\right)$ were seeded on Matrigel-coated wells of a 96-well plate with or without peptides for $12 \mathrm{~h}$. Tube formation was quantified by measuring tube numbers and total tube lengths in four random $\times 3$ magnification fields per well using a real-time cell history recorder (JuLi stage; NanoEnTek, Seoul, South Korea). The data analysis was performed using the Angiogenesis Analyzer in ImageJ (NIH).

\section{Immunoblot analysis}

Brain homogenates or whole-cell lysates were extracted with RIPA buffer ( $50 \mathrm{~mm}$ Tris- $\mathrm{HCl}$ ( $\mathrm{pH}$ 7.4), $150 \mathrm{~mm} \mathrm{NaCl,} 1 \mathrm{~mm}$ EDTA, $0.5 \%$ NP40, $0.25 \%$ sodium deoxycholate, $0.5 \%$ Triton X-100, $10 \%$ glycerol and Complete Mini Protease Inhibitor Cocktail tablet (Roche Diagnostics, Basel, Switzerland)), and protein concentrations in extracts were determined using a bicinchoninic acid assay. Cell or tissue extracts were then loaded into $8-10 \%$ SDS-PAGE gels and immunoblotted using the following primary antibodies: anti-vascular endothelial growth factor (VEGF) (1:3000; Abcam, Cambridge, UK), anti- $\alpha$-smooth muscle actin (1:500; Santa Cruz Biotechnology, Dallas, TX, USA), anti-matrix metallopeptidase 9 (MMP9) (1:3000, GeneTex, Irvine, CA, USA), anti-endothelial NOS (eNOS) (1:3000; Santa Cruz Biotechnology), anti-phospho-eNOS (1:3000; Cell Signaling Technology, Danvers, MA, USA), anti-neuronal NOS (nNOS) (1:3000; Santa Cruz Biotechnology), anti-phospho-nNOS (1:3000; Cell Signaling Technology), anti-integrin $\alpha_{4}$, anti-integrin $\alpha_{9}$, anti-integrin $\alpha_{v}$, antiintegrin- $\beta_{3}$ (1:2000; Santa Cruz Biotechnology), anti-phosphatidylinositol-4,5-bisphosphate 3-kinase (PI3K), anti-phospho-PI3K, anti-ERK, anti-phospho-ERK, anti-Akt, anti-phospho-Akt (1:3000; Cell Signaling) or anti- $\beta$-actin (1:4000; Applied Biological Materials, Richmond, BC, Canada) antibody. Blots were detected using anti-rabbit HP-conjugated or anti-mouse HP secondary antibody (1:3000, Millipore, Billerica, MA, USA) and a chemiluminescence kit (Thermo Fisher Scientific, Waltham, MA, USA).

\section{Preparation of conditioned media}

Cells were grown to subconfluency in gelatin-coated 12-well plates, starved for $12 \mathrm{~h}$ and then treated with OPNpt20 (1 $\mu \mathrm{M})$ or OPNpt20$\mathrm{Db}(1 \mu \mathrm{M})$ for $12 \mathrm{~h}$ with or without anti- $\alpha_{\mathrm{v}} \beta_{3}$ antibody $\left(0.1 \mu \mathrm{g} \mathrm{ml}^{-1}\right)$ or immunoglobulin $\mathrm{G}(\mathrm{IgG})\left(0.1 \mu \mathrm{g} \mathrm{ml}^{-1}\right)$. Conditioned medium was harvested and centrifuged at 8000 r.p.m. for $2 \mathrm{~min}$ at room temperature to eliminate dead cells. Supernatants were then transferred to clean tubes, and proteins were measured by immunoblotting and gelatin zymography.

\section{Pull-down assay}

Pull-down assays were performed using streptavidin agarose beads (Pierce, Rockford, IL, USA). Briefly, HUVEC lysates were preincubated with anti- $\alpha_{v} \beta_{3}$ antibody (Abcam) or IgG (Santa Cruz Biotechnology) for $15 \mathrm{~min}$ at $4{ }^{\circ} \mathrm{C}$ with rotation and then incubated with biotinylated-OPNpt20 (bt-OPNpt20, $0.1 \mu \mathrm{g} \mu \mathrm{l}^{-1}$ ) or biotinylatedOPNpt20-RAA (bt-OPNpt20-RAA, $0.1 \mu \mathrm{g} \mathrm{\mu l}^{-1}$ ) for $30 \mathrm{~min}$. These mixtures were incubated with $20 \mu \mathrm{l}$ of streptavidin beads for $30 \mathrm{~min}$ at $4{ }^{\circ} \mathrm{C}$, centrifuged at 8000 r.p.m. for $2 \mathrm{~min}$, washed three times and analyzed by immunoblot using anti-integrin $\alpha \mathrm{V}$ (1:1000; Santa Cruz Biotechnology) and anti- $\beta$-actin (1:4000; Applied Biological Materials) antibodies.

\section{Gelatin zymography}

Conditioned medium from HUVEC cultures was adjusted to the same protein concentration and analyzed for gelatin digestion by 
a

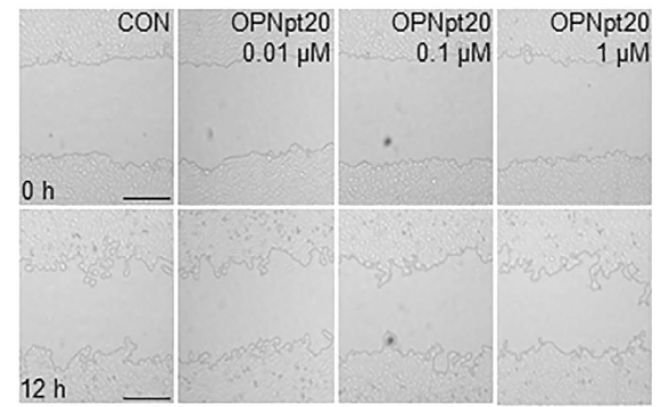

b

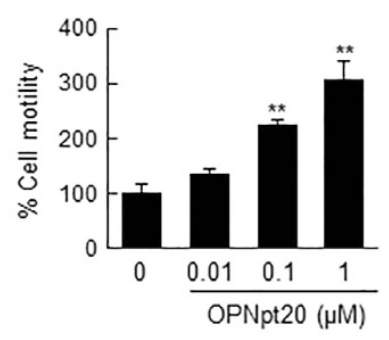

d

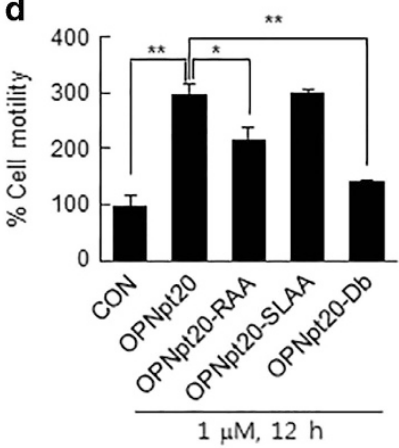

Figure 2 Induction of cell migration by 20-amino-acid OPN peptide (OPNpt20) and its mutants in human umbilical vein endothelial cell (HUVEC) cultures. (a, b) Cell migration was evaluated using a wound healing assay in HUVEC cultures after incubation with OPNpt20 $(0.01,0.1$ or $1 \mu \mathrm{m})$ for $12 \mathrm{~h}$. (b) Cell motility was assessed by measuring wound widths at 0 and $12 \mathrm{~h}$. The results are presented as the mean \pm s.e.m. $(n=4)$. (c, d) HUVEC migration was measured after treatment with the three mutant peptides $(1 \mu \mathrm{m})$ for $12 \mathrm{~h}$. Representative images are presented $(\mathbf{a}, \mathbf{c})$, and results are presented as the mean \pm s.e.m. $(n=3)(\mathbf{b}, \mathbf{d})$. Scale bars, $400 \mu \mathrm{m}$. ${ }^{*} P<0.05$, ${ }^{*} P<0.01$ between the indicated groups.

electrophoresis under non-reducing conditions on SDS-polyacrylamide gels containing gelatin $\left(1 \mathrm{mg} \mathrm{ml}^{-1}\right)$. Gels were washed with washing buffer (50 mм Tris- $\mathrm{HCl}(\mathrm{pH} 7.5), 2.5 \%$ Triton X-100, $5 \mathrm{mM} \mathrm{CaCl}_{2}, 1 \mu \mathrm{M} \mathrm{ZnCl}_{2}$ ) to remove SDS from gels and, $30 \mathrm{~min}$ later, were incubated in incubation buffer (50 mM Tris- $\mathrm{HCl}(\mathrm{pH} 7.5), 1 \%$ Triton X-100, $5 \mathrm{~mm} \mathrm{CaCl}, 1 \mu \mathrm{M} \mathrm{ZnCl}_{2}$ ) for $72 \mathrm{~h}$ at $37^{\circ} \mathrm{C}$. Gels were stained with staining solution ( $0.5 \%$ Coomassie brilliant blue, $40 \%$ methanol, $10 \%$ acetic acid) for $1 \mathrm{~h}$ at room temperature and then incubated with destaining solution (40\% methanol, $10 \%$ acetic acid) until white degraded zones were clearly seen.

\section{Immunohistochemistry}

Brains were fixed in $4 \%$ paraformaldehyde solution for 2 days at $4{ }^{\circ} \mathrm{C}$ and postfixed in $30 \%$ sucrose solution at $4{ }^{\circ} \mathrm{C}$. Brain sections were obtained by sectioning at $30 \mu \mathrm{m}$ using a vibratome, and immunological staining was performed. Sections were then blocked with $5 \%$ FBS, $5 \%$ horse serum and $2 \%$ albumin in $0.1 \%$ Triton X-100 for $1 \mathrm{~h}$ at room temperature. Primary antibodies anti-rat endothelial cell antigen-1 (RECA-1) (AbD Serotec, Kidlington, UK) and anti- $\alpha-$ smooth muscle actin (Santa Cruz Biotechnology) were used at a concentration of 1:500. After incubation with primary antibodies, brain sections were washed with PBS and incubated with rhodaminelabeled anti-rabbit IgG (1:300; Jackson ImmunoRes, West Grove, PA) secondary antibody. Sections were then mounted with mounting solution containing DAPI (Vector Laboratories, Peterborough, UK), and endothelial areas were analyzed using the AngioTool Software (National Cancer Institute, Gaithersburg, MD, USA).

\section{Tissue preparation and immunohistochemistry for} hypoxyprobe adducts

To detect hypoxic areas in brain, rats were injected intraperitoneally with hypoxyprobeTM-1 (60 $\mathrm{mg} \mathrm{kg}^{-1}$, solid pimonidazole hydrochloride; Natural Pharmacia International, Burlington, MA, USA) at $3 \mathrm{~h}$ before being killed. Isolated brains were immediately immersed in $4 \%$ paraformaldehyde for 2 days at $4{ }^{\circ} \mathrm{C}$ and postfixed in $30 \%$ sucrose solution at $4{ }^{\circ} \mathrm{C}$. Brains were then sectioned and stained with fluorescein isothiocyanate -conjugated anti-pimonidazole antibody.

\section{Statistical analysis}

Statistical analysis was performed using the two-tailed Student's $t$-test. The results are presented as the mean \pm s.e.m., and statistical significance was accepted for $P$-values $<0.05$.

\section{RESULTS}

OPNpt20 induced HUVEC proliferation, and its RGD and SLAY motifs had critical roles

Angiogenesis is a complex biological process that requires the precise coordination of proliferation, migration and tube formation by endothelial cells. To determine whether OPNpt20 (Figure 1a) induces endothelial cell proliferation, HUVECs were treated with $0.01,0.1$ or $1 \mu \mathrm{M}$ of OPNpt 20 for $24 \mathrm{~h}$, and the total number of Ki67-positive cells were counted to measure cell proliferation. Ki67-positive cell numbers were found to increase in an OPNpt20 dose-dependent manner, and $1 \mu \mathrm{M}$ of OPNpt 20 induced a 4.8 -fold increase 
a

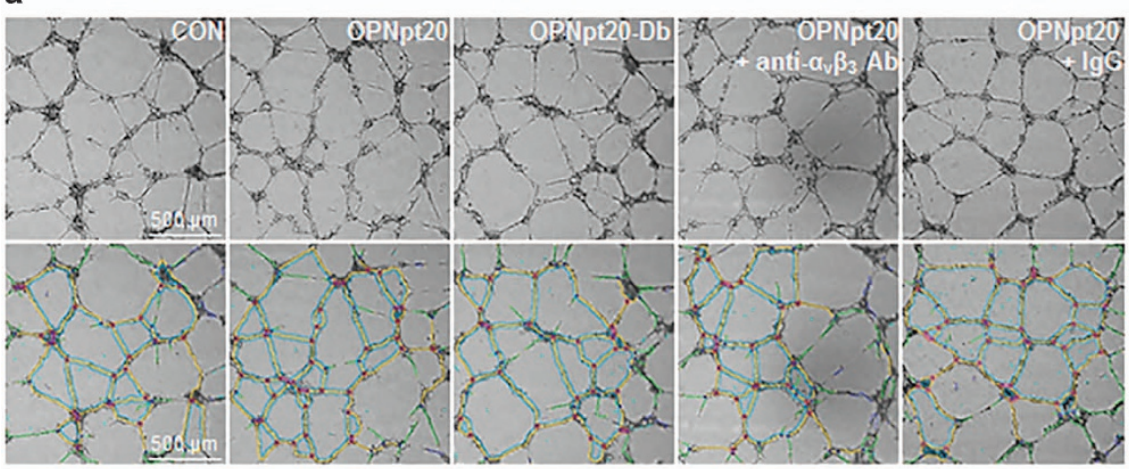

b

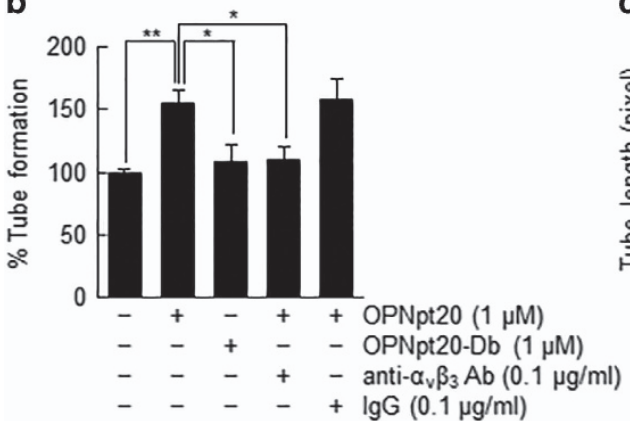

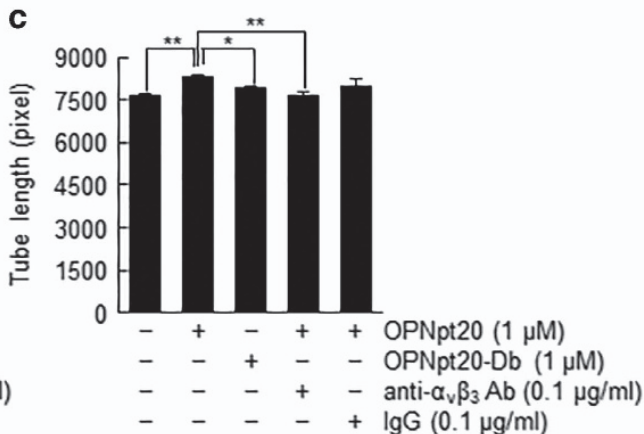

Figure 3 Induction of tube formation by 20-amino-acid OPN peptide (OPNpt20) in human umbilical vein endothelial cell (HUVEC) cultures. OPNpt20 (1 $\mu \mathrm{m})$-induced tube formation was examined in HUVECs treated with OPNpt20 (1 $\mu \mathrm{m})$ or OPNpt20-Db (mutant OPN peptide with both RGD and SLAY replaced; $1 \mu \mathrm{m}$ ) for $12 \mathrm{~h}$ in the presence or absence of anti- $\alpha_{\Downarrow} \beta_{3}$ antibody or immunoglobulin $\mathrm{G}$ (IgG). Representative images obtained from Image J analyzer (National Institute of Health, Bethesda, MD, USA) are presented (green, branches; yellow, master segments; blue, tubes; red, master junctions) (a). Tube numbers were counted (b), and tube lengths were measured (c). The results are presented as the mean \pm s.e.m. $(n=3)$. Scale bars, $500 \mu \mathrm{m}$. ${ }^{*} P<0.05,{ }^{*} P<0.01$ between the indicated groups.

versus treatment-naïve controls (Figure $1 \mathrm{~b}$ and $\mathrm{c}$ ). To examine the involvement of the RGD and SLAY motifs of OPNpt20 in HUVEC proliferation, cells were treated with $1 \mu \mathrm{M}$ of three different mutant OPNpt20 peptides (Figure 1a), in which RGD was replaced with RAA (OPNpt20-RAA), SLAY was replaced with SLAA (OPNpt20-SLAA) or RGDSLAY was replaced with RAASLAA (OPNpt20-Db), for $24 \mathrm{~h}$. HUVEC proliferation was reduced to $63.0 \pm 5.7 \%$ or $26.8 \pm 4.2 \%$ in OPNpt 20 -RAAand OPNpt20-Db-treated cells, respectively, of that in OPNpt20-treated cells but not in OPNpt20-SLAA-treated cells (Figure 1e and f). OPNpt20-mediated HUVEC proliferation was confirmed by the MTT assay, and compared with OPNpt20-treated cells, the results showed that cell survival was lower for OPNpt20-RAA- and OPNpt20-Db-treated cells but not for OPNpt20-SLAA-treated cells (Figure 1d and g). These results indicate that OPNpt20 induced HUVEC proliferation, that the RGD motif of OPNpt20 has a critical role and that the SLAY motif also contributes to this process but to a lesser extent.

OPNpt20 induced HUVEC migration in an RGD and SLAY motif-dependent manner

To determine whether OPNpt20 induces HUVEC migration, we used a wound healing assay after treating cells with OPNpt20 $(0.01,0.1$ or $1 \mu \mathrm{M})$ or its three mutant peptides $(1 \mu \mathrm{M})$ for $12 \mathrm{~h}$. Cell motility was determined by measuring wound widths and was found to increase in a dose-dependent manner in OPNpt20-treated cells (Figure 2a and b). At $1 \mu \mathrm{M}$ of OPNpt20, cell migration increased to $299.6 \pm 16.7 \%$ of that of treatment-naïve controls (Figure $2 \mathrm{c}$ and $\mathrm{d}$ ). However, cell migration in OPNpt20-RAA- and OPNpt20-Db-treated cells was reduced to $72.9 \pm 6.4 \%$ and $47.0 \pm 1.2 \%$, respectively, of that in OPNpt20-treated cells, while cell migration in OPNpt20-SLAA-treated cells was comparable to that in OPNpt20-treated cells (Figure $2 \mathrm{c}$ and $\mathrm{d}$ ). These results indicate that OPNpt20 induced HUVEC migration, its RGD motif had a critical role and its SLAY motif had a lesser role.

\section{OPNpt20 induced tube formation by HUVECs in an endogenous $\alpha_{v} \beta_{3}$-integrin-dependent manner}

To confirm the proangiogenic effects of OPNpt20, we examined tube formation by HUVECs. Tube-like structures harboring branches, segments and nodes were observed after culturing HUVECs on Matrigel for $12 \mathrm{~h}$ (Figure 3a). OPNpt20 $(1 \mu \mathrm{M})$ increased tube formation to $155.3 \pm 9.4 \%$ versus treatment-naïve control cells, but treatment with OPNpt20-Db did not enhance tube formation (Figure $3 \mathrm{a}$ and $\mathrm{b}$ ). Interestingly, tube formation did not increase when anti- $\alpha_{v} \beta_{3}$-integrin antibody was cotreated with OPNpt20 (OPNpt20+anti- $\alpha_{v} \beta_{3}$ antibody), but increased when IgG was cotreated (OPNpt20+IgG) (Figure $3 \mathrm{a}$ and $\mathrm{b}$ ), indicating that $\alpha_{\mathrm{v}} \beta_{3}$-integrin is critical for OPNpt20-mediated tube formation. Similarly, measurements 

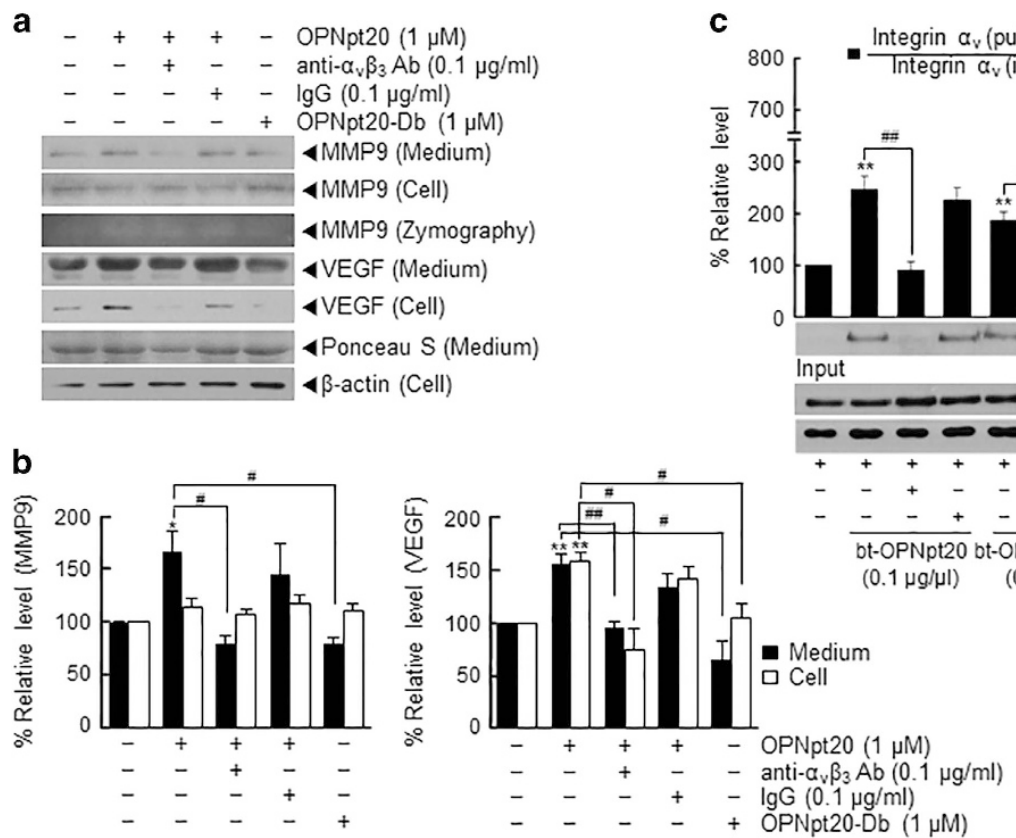

Figure 4 Endogenous $\alpha_{v} \beta_{3}$-integrin mediates the proangiogenic effect of 20-amino-acid OPN peptide (OPNpt20) in human umbilical vein endothelial cells (HUVECs). (a, b) Protein levels of matrix metallopeptidase 9 (MMP9) and vascular endothelial growth factor (VEGF) in conditioned media or HUVEC lysates were assessed by immunoblotting after treatment with OPNpt20 ( $1 \mu \mathrm{m}$ ) or OPNpt20-Db (mutant OPN peptide with both RGD and SLAY replaced; $1 \mu \mathrm{m})$ for $12 \mathrm{~h}$ in the presence or absence of anti- $\alpha_{v} \beta_{3}\left(0.1 \mu \mathrm{g} \mathrm{ml}{ }^{-1}\right)$ antibody or IgG $\left(0.1 \mu \mathrm{g} \mathrm{ml}^{-1}\right)$. The results are presented as the mean \pm s.e.m. $(n=3)$ (b). (c) Direct binding between endogenous integrin $\alpha_{v}$ and biotinylated-OPNpt20 or biotinylated-OPNpt20-RAA was examined using a pull-down assay. HUVEC lysates (2.5 $\mu \mathrm{g}^{-1}$ ) were preincubated with anti- $\alpha_{v} \beta_{3}$ or IgG $\left(0.5 \mathrm{ng} \mu^{-1}\right)$ for $15 \mathrm{~min}$ and then incubated with biotinylated-OPNpt20 or biotinylated-OPNpt20-RAA for $30 \mathrm{~min}$. Complexes were pulled down with streptavidin beads, and $\alpha_{v}$-integrin was measured by immunoblot using anti- $\alpha_{v}$-integrin antibody. Representative images are presented and results are presented as the mean \pm s.e.m. $(n=4)\left(\right.$ c). ${ }^{*} P<0.05,{ }^{*} P<0.01$ versus treatment-naïve controls, ${ }^{\#} P<0.05,{ }^{\#} P<0.01, \$ \$ P<0.01$ between the indicated groups.

taken after culturing HUVECs on Matrigel for $12 \mathrm{~h}$ revealed that total tube lengths were also significantly increased in the presence of the RGDSLAY motif and $\alpha_{v} \beta_{3}$-integrin, which confirmed the notion that endogenous $\alpha_{v} \beta_{3}$-integrin is required for OPNpt20-mediated tube formation (Figure $3 a$ and c).

The interaction between OPNpt20 and endogenous $\boldsymbol{\alpha}_{\mathrm{v}} \boldsymbol{\beta}_{3^{-}}$ integrin mediated the proangiogenic effect of OPNpt20 in HUVECs

Protein levels of MMP9 and VEGF (proangiogenic markers) in media were higher for OPNpt20-treated HUVECs than for treatment-naïve controls (Figure 4a and b). Conversely, accumulation of MMP9 and VEGF in OPNpt20-treated HUVEC medium was significantly reduced by cotreating cells with anti$\alpha_{v} \beta_{3}$ antibody but not by cotreating with IgG (Figure $4 a$ and b), indicating that the interaction between OPNpt20 and $\alpha_{\mathrm{v}} \beta_{3^{-}}$ integrin also has an important role in the induction of MMP9 or VEGF in HUVECs. Induction of MMP9 was confirmed with zymography (Figure 4a). As expected, OPNpt20-Db failed to induce upregulation of MMP9 and VEGF (Figure 4a and b). Furthermore, when a pull-down assay was performed using bt-OPNpt20, an interaction was detected between bt-OPNpt20 and endogenous integrin $\alpha_{\mathrm{v}}$ in HUVEC lysates. However, this interaction was not detected when HUVEC lysates was preincubated with anti- $\alpha_{\mathrm{v}} \beta_{3}$-integrin antibody (Figure $4 \mathrm{c}$ ).
This interaction with endogenous integrin $\alpha_{\mathrm{v}}$ was also detected when bt-OPNpt20-RAA was used instead of bt-OPNpt20, but not after preincubating HUVEC lysates with anti- $\alpha_{v} \beta_{3}$ integrin antibody (Figure 4c). These results show that exogenous OPNpt20 and endogenous $\alpha_{v} \beta_{3}$ integrin interact, and this interaction might be responsible for the proangiogenic effect of OPNpt20 in HUVECs.

\section{$\boldsymbol{\alpha}_{v} \boldsymbol{\beta}_{3}$-Integrin/AKT and ERK signaling pathways were} involved in OPNpt20-mediated angiogenesis

PI3K, AKT and ERK signaling pathways are involved in OPN-induced angiogenesis in HUVEC cultures. ${ }^{18}$ We found that in OPNpt20-treated HUVEC cultures, ERK and AKT were phosphorylated within $30 \mathrm{~min}$ of treatment (Figure 5a), and maximum phosphorylation occurred at OPNpt20 concentrations of 0.1 or $1 \mu \mathrm{M}$, respectively (Figure 5b). Furthermore, phosphorylation of ERK and AKT was suppressed by pretreating cells with anti- $\alpha_{v} \beta_{3}$ antibody (100 $\mathrm{ng} \mathrm{ml}^{-1}$ ) for $30 \mathrm{~min}$ but not by pretreating cells with $\operatorname{IgG}\left(100 \mathrm{ng} \mathrm{ml}^{-1}, 30 \mathrm{~min}\right)$ (Figure 5c). The phosphorylation of ERK and AKT was also observed in OPNpt20-RAA- or OPNpt20-SLAA-treated cells and suppressed by pretreating with anti- $\alpha_{v} \beta_{3}$ antibody (Figure 5c). However, the phosphorylation of ERK and AKT were not detected in OPNpt20-Db-treated cells. Taken together, these results indicated that endogenous $\alpha_{v} \beta_{3}$-integrin 
a

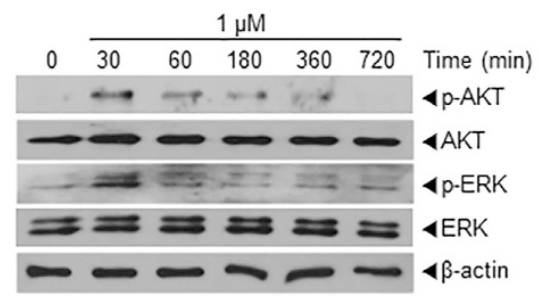

b

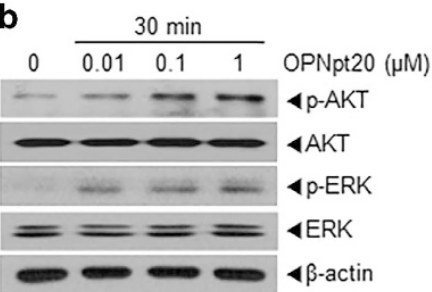

c
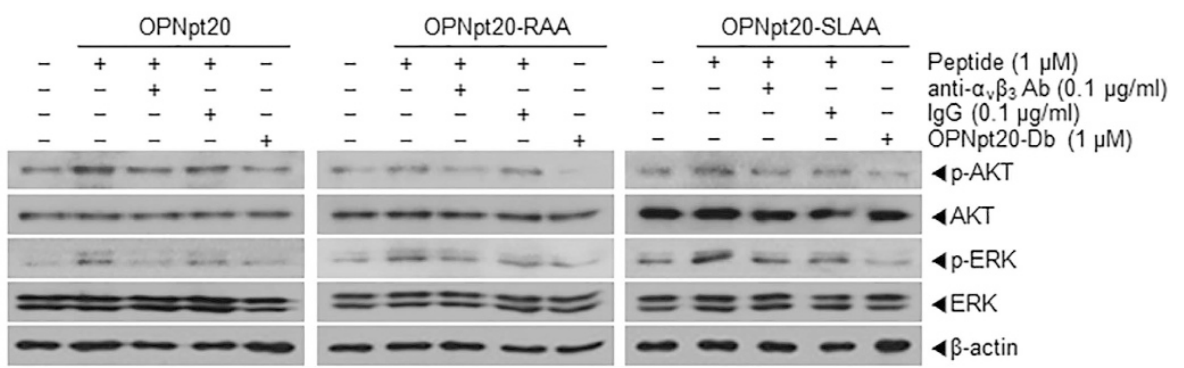

- lgG $a_{v} \beta_{3} A b(0.1 \mu$

- $\operatorname{lgG}(0.1 \mu \mathrm{\mu g} / \mathrm{ml})$

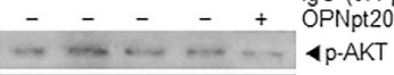

$-\infty-\infty \mathrm{AKT}$

$--\ldots-\ldots$ 4.ERK

$== \pm= \pm \mathrm{ERK}$
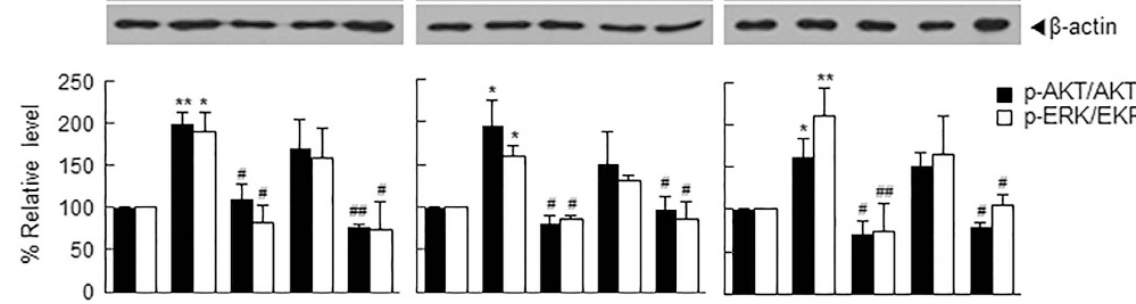

Figure 5 Activation of ERK and AKT pathways by 20-amino-acid OPN peptide (OPNpt20) in human umbilical vein endothelial cellS (HUVECs) and the role played by $\alpha_{v} \beta_{3}$-integrin. (a, b) HUVECs were incubated with OPNpt20 (1 $\left.\mu \mathrm{m}\right)$ for 30, 60, 180 or 360 min (a) or with $0.01,0.1$ or $1 \mu \mathrm{m}$ of OPNpt20 for $1 \mathrm{~h}(\mathbf{b})$, and total and phosphorylated-ERK and -AKT levels were assessed by immunoblotting. (c) HUVECs were incubated with OPNpt20 $(1 \mu \mathrm{m})$ or its three mutant peptides for 30 min in the presence or absence of anti- $\alpha_{v} \beta_{3}$ antibody or IgG, and total and phosphorylated-ERK and -AKT levels were assessed by immunoblotting. Representative images are presented, and results are presented as the mean \pm s.e.m. $(n=3)$. ${ }^{*} P<0.05,{ }^{* *} P<0.01$ versus treatment-naïve controls, ${ }^{\#} P<0.05$, \#\# $P<0.01$ versus OPNpt20-, OPNpt20-RAA or OPNpt20-SLAA-treated cells.

interacts with the RGD and SLAY motifs of OPNpt20 and that these interactions are important for activating AKT and ERK signaling pathways in HUVECs.

Proangiogenic effects of OPNpt20 in the postischemic brain We also examined whether OPNpt20 exerts proangiogenic effects in the postischemic brain using a rat MCAO model. To confirm that OPNpt20 has a direct proangiogenic effect rather than an indirect effect caused by its neuroprotective effects, OPNpt20 ( $1.7 \mu \mathrm{g} / 100 \mathrm{~g}$ body weight $)$ was administered intranasally at 4, 5 and 6 days after MCAO, and vessel formation was examined at 7 days after MCAO (Figure 6a). Infarct volumes in the OPNpt20- and OPNpt20-Db-administered MCAO groups were similar to those in PBS-administered MCAO controls, as determined by cresyl violet staining at 7 days after MCAO (Figure 6b). Blood vessel densities in cortical penumbra of ipsilateral hemispheres (Figure 6b, asterisk) were measured by immunostaining using anti- RECA-1 (a marker of endothelial cells) antibody. RECA-1-positive vessel density was slightly higher in treatment-naïve $\mathrm{MCAO}$ controls than in sham controls at 7 days after MCAO, and these densities were significantly enhanced in the OPNpt20administered MCAO animals $(160.8 \pm 12.0 \%$ of that in treatment-naïve MCAO controls) (Figure $6 \mathrm{c}$ and $\mathrm{d}$ ). However, OPNpt20-Db did not increase vessel density (Figure $6 \mathrm{c}$ and d).
Similarly, total vessel length was markedly increased in the OPNpt20-administered MCAO animals $(226.1 \pm 11.2 \%$ of treatment-naïve MCAO controls), but not in the OPNpt20-Db animal (Figure 6e). Taken together, these results demonstrate a robust proangiogenic effect of OPNpt20 in the postischemic brain. To determine whether OPNpt20 induces functional blood vessels, hypoxia levels in the cortical penumbra of the ipsilateral hemisphere (asterisk in Figure 6a) were measured using a hypoxyprobe (pimonidazole) (Figure 6a). We found that hypoxia was significantly lower in OPNpt20-administered MCAO animals, $42.7 \pm 8.1 \%$ of that in PBS-treated MCAO controls (Figure $6 \mathrm{f}$ and $\mathrm{g}$ ), indicating that the new vessels functioned properly. In addition, levels of angiogenesisassociated proteins, such as VEGF and $\alpha$-smooth muscle actin, were also found to be upregulated in the same brain areas in the OPNpt20-administered group at 7 days after MCAO, but not in the OPNpt20-Db-administered MCAO group (Figure 6h). Furthermore, eNOS phosphorylation (Ser1177), which was induced in the postischemic brain, was further enhanced by OPNpt20, and similar enhancement of phosphonNOS (Ser 1417) induction was detected in OPNpt20-treated animals (Figure 6i). These results confirm the proangiogenic potency of OPNpt20 in the postischemic brain and the importance of the contributions made by RGD and SLAY. 


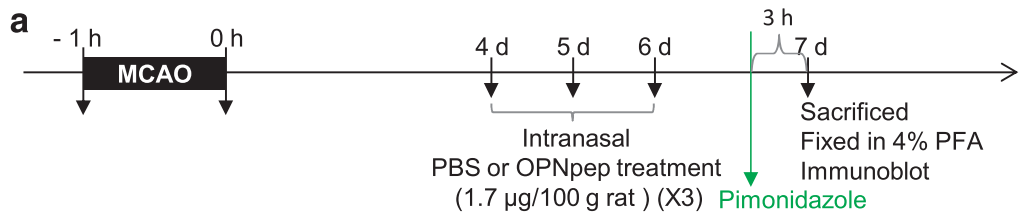

b
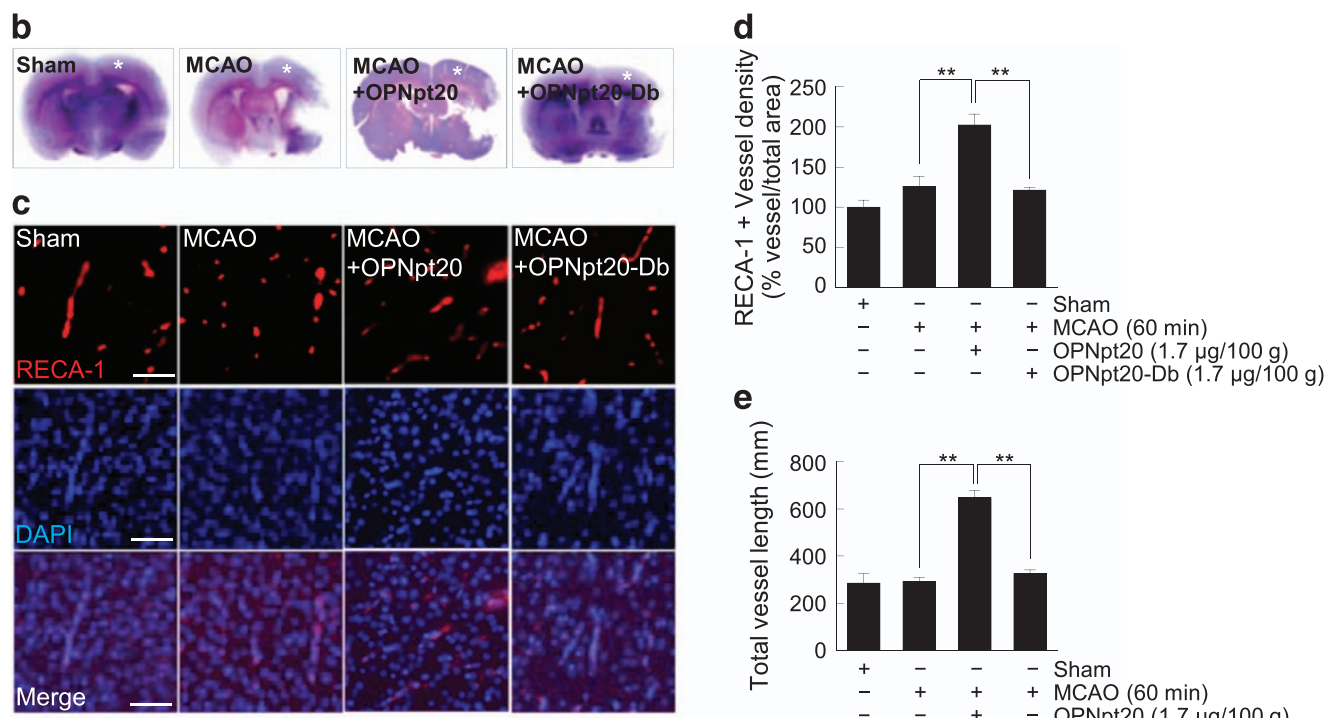

f
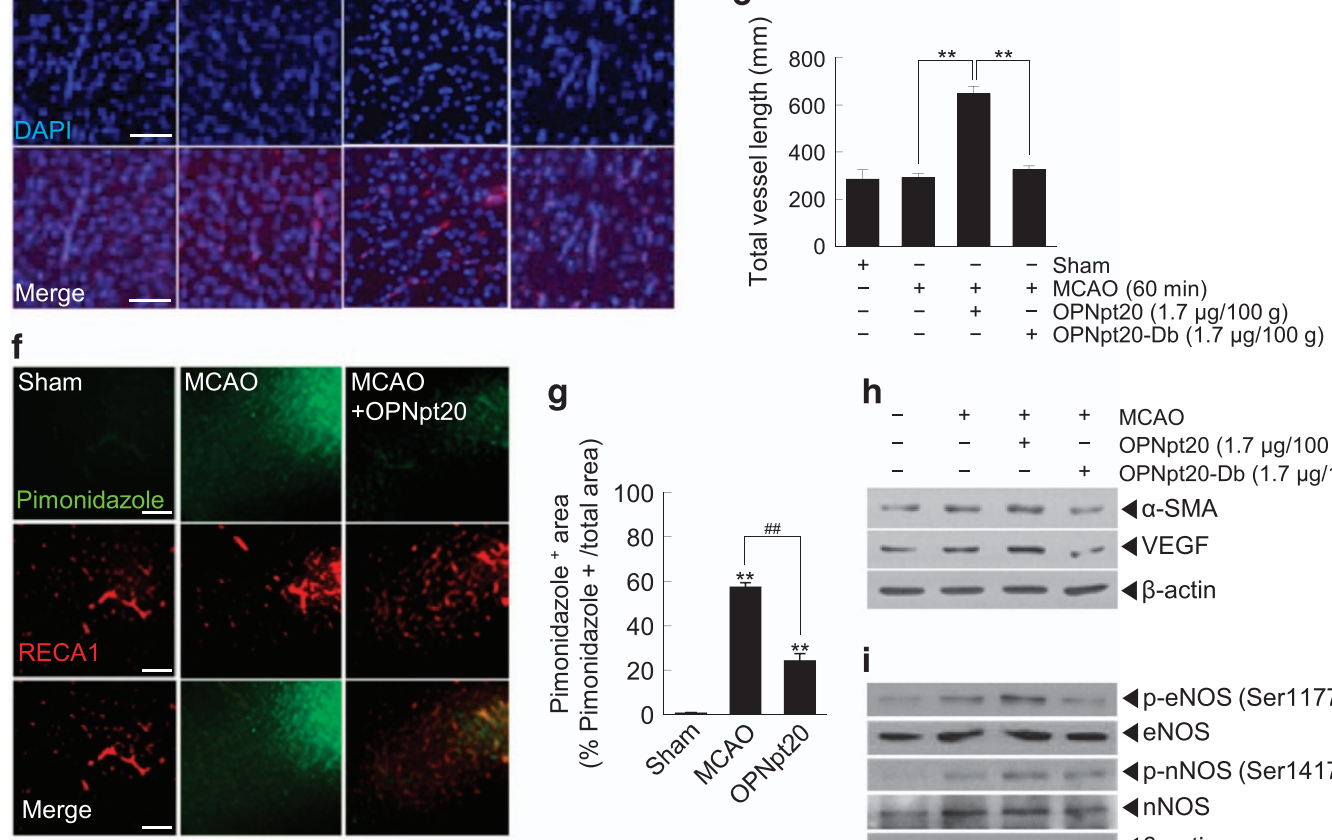

h

-+++ MCAO

$-\quad-\quad+\quad-$ OPNpt20 $(1.7 \mu \mathrm{g} / 100 \mathrm{~g})$

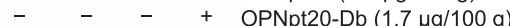

- - - $4 \mathrm{\alpha}-\mathrm{SMA}$
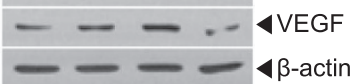

i

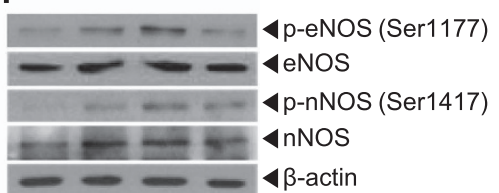

Figure 6 Induction of angiogenesis and of proangiogenic markers by 20-amino-acid OPN peptide (OPNpt20) in postischemic brains. (a) Schematic of the experiment. (b-e) OPNpt20 or OPNpt20-Db (mutant OPN peptide with both RGD and SLAY replaced; $1.7 \mu \mathrm{g} / 100 \mathrm{~g}$ body weight) were administered intranasally three times at 4, 5 and 6 days after 60 min of middle cerebral artery occlusion (MCAO). Seven days after MCAO, coronal brain sections were stained using cresyl violet (b) or double fluorescence-stained with anti-rat endothelial cell antigen-1 (RECA-1) antibody and DAPI (4',6-diamidino-2-phenylindole) (c). Asterisks in (b) indicated areas examined in double fluorescent staining. Representative images are presented (c), and RECA-1-positive vessel densities are presented as the mean \pm s.e.m. $(n=5)(\mathbf{d})$. (e) Total vessel length was measured using the AngioTool Software (National Cancer Institute, Gaithersburg, MD, USA), and results are presented as the mean \pm s.e.m. $(n=5)$. Scale bars, $250 \mu \mathrm{m}$. ${ }^{*} P<0.01$ between the indicated groups. (f, g) OPNpt20 (1.7 $\mu \mathrm{g} / 100 \mathrm{~g}$ body weight) was administered intranasally three times at 4,5 and 6 days after $60 \mathrm{~min}$ of MCAO and hypoxyprobe (pimonidazole, 60 mg kg ${ }^{-1}$ ) was injected (intraperitoneally (i.p.)) $3 \mathrm{~h}$ before killing at 7 days after MCAO (a). Coronal brain sections were prepared and immunostained with fluorescein isothiocyanate (FITC)-conjugated anti-pimonidazole antibody. Representative images are presented (f), and results are presented as the mean \pm s.e.m. $(n=6)(\mathbf{g})$. (h, i) Tissue lysates were prepared from asterisked regions in (b) at 7 days after MCAO and immunoblotted for vascular endothelial growth factor (VEGF), $\alpha$-smooth muscle actin (SMA), total or phosphorylated endothelial NOS (Enos), total or phosphorylated neuronal NOS (nNOS) and $\beta$-actin. The results are representative of three independent experiments.

\section{DISCUSSION}

In a previous study, we reported that intranasally delivered OPNpt20 had a robust neuroprotective effect in the postischemic brain because of an anti-inflammatory effect mediated by the interaction between the RGD motif of OPNpt20 and $\alpha_{v} \beta_{3}$-integrin. ${ }^{16}$ In the present study, we found that OPNpt20 has in vitro and in vivo proangiogenic effects. Although the proangiogenic effect of OPN has been documented under various pathophysiological conditions, including in various cancers, ${ }^{19,20}$ the results obtained in the present study show 
that the exogenous OPN icosamer peptide, OPNpt20, binds to endogenous $\alpha_{\mathrm{v}} \beta_{3}$-integrin and induces proangiogenic effects in vitro and in the postischemic brain using mutant peptides and blocking antibodies.

In the present study, we found that the RGD motif has a critical role and that the SLAY motif also contributes to a lesser degree to the proangiogenic effect of OPNpt20, which is in keeping with previous reports showing that the RGD motif of OPN is essential for OPN-induced angiogenesis. It has been reported that an $\mathrm{N}$-terminal fragment of OPN containing its RGD motif promoted adhesion of mouse and human fibroblasts more effectively than full-length OPN.$^{21}$ In addition, cell attachment and spreading stimulated by an $\mathrm{N}$-terminal fragment of OPN was inhibited by soluble GRGDS peptides or an OPN-specific antibody raised against the GRGDS domain of OPN. ${ }^{22}$ Furthermore, recent reports have shown that OPN and integrin has essential roles in angiogenesis in many tumor types. ${ }^{19,20}$ In the present study, we showed that the RGD motif of OPNpt20 interacts with endogenous $\alpha_{v} \beta_{3}$-integrin and that this interaction is important for activating AKT and ERK signaling pathways and subsequent angiogenic processes, such as tube formation, in HUVECs.

In terms of the SLAY motif of OPN, the proangiogenic activity of the SVVYGLR peptide has been reported in transformed rat lung endothelial cells, ${ }^{23}$ in a dorsal air sac model, ${ }^{24}$ in animal models of heart failure ${ }^{25}$ and in myocardial fibrosis. ${ }^{26}$ Furthermore, the SLAY motif has been reported to bind to various integrins, such as $\alpha_{v} \beta_{3}, \alpha_{4} \beta_{1}, \alpha_{4} \beta_{7}$ or $\alpha_{9} \beta_{1}$, in a cell-type-dependent manner but HUVEC. ${ }^{11,12,27,28}$ As the SLAY motif is located adjacent to the RGD sequence, the RGD and SLAY motifs in OPNpt20 appear to compete for binding with $\alpha_{v} \beta_{3}$-integrin. However, in the present study, OPNpt20-RAA was found to have greater angiogenic potency than OPNpt20$\mathrm{Db}$, but comparable potency was observed for OPNpt20-SLAA and OPNpt20 (Figures 1 and 2). Based on these observations, we speculate that the RGD motif binds preferentially with $\alpha_{v} \beta_{3}$-integrin and thus has a critical role, but in the absence of the RGD motif, the SLAY motif has a proangiogenic role. Furthermore, the possibility that integrins other than $\alpha_{v} \beta_{3}$ bind to these two motifs cannot be excluded, and this possibility needs to be further explored.

In the present study, OPNpt20 conferred proangiogenic effects by activating the ERK and AKT signaling pathways (Figure 5), which is in accordance with a previous report regarding the involvement of ERK and AKT in OPN-mediated angiogenesis. ${ }^{18}$ Significant reductions in phosphorylated-ERK and phosphorylated-AKT levels after treatment with $\alpha_{v} \beta_{3}$-integrin antibody in OPNpt20-, OPNpt20-RAA- and OPNpt20-SLAA-treated cells (Figure $5 \mathrm{c}$ ) indicated that these signaling pathways are used in both RGD- and SLAY-mediated angiogenic processes. Furthermore, since a positive feedback loop has been reported between OPN-mediated VEGF induction and VEGF-mediated OPN induction, in which the PI3K/AKT and ERK pathways has important roles, ${ }^{18}$ it is possible that OPNpt20 might be able to trigger these positive feedback signals. In addition, since the phosphorylation of Ser 1177 or Ser 1417 of eNOS and nNOS, respectively, have been suggested to attenuate ischemic injury, ${ }^{29,30}$ marked induction and activation of eNOS and nNOS by OPNpt20 might underlie its proangiogenic effects.

In the postischemic brain, angiogenesis at 7 days after MCAO was significantly induced when OPNpt20 was administered at 4,5 and 6 days after MCAO (Figure 6), which was probably too late to affect infarct volumes. This suggested that OPNpt20 induced angiogenesis independent of its neuroprotective effects. Interestingly, OPN is induced in a delayed manner in various animal models of stroke. For example, in a mouse model of permanent focal ischemia, OPN induction began at $12 \mathrm{~h}$ and peaked at 5 days, and in a rat model of transient forebrain ischemia, induction began at $6 \mathrm{~h}$ and peaked at 5 days. ${ }^{8}$ In addition, delayed induction of $\alpha_{v} \beta_{3}$-integrin in peri-infarct astrocytes after 5 and 15 days has been reported in a postpermanent rat model of $\mathrm{MCA},{ }^{31}$ which suggests that OPN-integrin interactions have important functions during the later period in the postischemic brain. This raises the possibility that administration of exogenous OPNpt20, which has effects comparable to OPN at earlier times, could accelerate repair processes in the postischemic brain. Furthermore, OPNpt20-mediated VEGF induction (Figures 4 and 6 and Supplementary Figure 1) might initiate a second round of proangiogenic processes. In terms of the efficiency of OPNpt20 delivery into the brain, we detected exogenously delivered fluorescein isothiocyanate-conjugated OPNpt20 in cerebral cortices 2 and $8 \mathrm{~h}$ after administration and its colocalization with endothelial cells and $\alpha_{\mathrm{v}}$-integrin (Supplementary Figure 2). Further studies are required to identify specific interactions with different cell types, brain regions and times.

Because OPN is a multifunctional protein, it is possible that OPNpt20 might have effects other than anti-inflammatory ${ }^{16}$ and proangiogenic effects, such as antiapoptotic effects, ${ }^{32}$ the scavenging of toxic substances like excess $\mathrm{Ca}^{2+}$ or dead neurons, ${ }^{33,34}$ or attractant/inducer functions that drive the migration of neuroblasts from the subventricular zone to ischemic regions. ${ }^{35}$ Although additional studies are required to determine whether OPNpt20 indeed performs the abovementioned functions, it offers great promise as a multifunctional therapeutic agent. Compared with whole OPN, the greater diffusion of OPNpt20 and its enhanced interaction with $\alpha_{v} \beta_{3}$-integrin, which is caused by mobilization of its $\mathrm{C}$ terminus, increase its proangiogenic potency. Furthermore, OPNpt20 is derived from an endogenous protein, reducing safety concerns.

\section{CONFLICT OF INTEREST}

The authors declare no conflict of interest.

\section{ACKNOWLEDGEMENTS}

This work was supported by a Mid-career Research Grant (NO. 2015R1A2A2A01003395) from the Korean National Research Foundation (to J-KL) and by a Medical Research Center Grant (No. 2014R1A5A2009392) from the Korean National Research Foundation (to J-KL). 


\section{PUBLISHER'S NOTE}

Springer Nature remains neutral with regard to jurisdictional claims in published maps and institutional affiliations.

1 Reinholt FP, Hultenby K, Oldberg A, Heinegård D. Osteopontin-a possible anchor of osteoclasts to bone. Proc Natl Acad Sci USA 1990; 87: 4473-4475.

2 Yasui T, Fujita K, Asai K, Kohri K. Osteopontin regulates adhesion of calcium oxalate crystals to renal epithelial cells. Int J Urol 2002; 9: $100-108$.

3 Isoda K, Nishikawa K, Kamezawa Y, Yoshida M, Kusuhara M, Moroi M et al. Osteopontin plays an important role in the development of medial thickening and neointimal formation. Circ Res 2002; 91: 77-82.

4 Shimizu Y, Ota K, Ikeguchi R, Kubo S, Kabasawa C, Uchiyama S. Plasma osteopontin levels are associated with disease activity in the patients with multiple sclerosis and neuromyelitis optica. J Neuroimmunol 2013; 263 : $148-151$.

5 Iwadate H, Kobayashi H, Kanno T, Asano T, Saito R, Sato S et al. Plasma osteopontin is correlated with bone resorption markers in rheumatoid arthritis patients. Int J Rheum Dis 2014; 17: 50-56.

6 Fedarko NS, Jain A, Karadag A, Van Eman MR, Fisher LW. Elevated serum bone sialoprotein and osteopontin in colon, breast, prostate, and lung cancer. Clin Cancer Res 2001; 7: 4060-4066.

7 Choi JS, Kim HY, Cha JH, Choi JY, Lee MY. Transient microglial and prolonged astroglial upregulation of osteopontin following transient forebrain ischemia in rats. Brain Res 2007; 1151: 195-202.

8 Wang X, Louden C, Yue TL, Ellison JA, Barone FC, Solleveld HA et al. Delayed expression of osteopontin after focal stroke in the rat. $J$ Neurosci 1998; 18: 2075-2083.

9 van Velthoven CT, Heijnen CJ, van Bel F, Kavelaars A. Osteopontin enhances endogenous repair after neonatal hypoxic-ischemic brain injury. Stroke 2011; 42: 2294-2301.

10 Rangaswami H, Bulbule A, Kundu GC. Nuclear factor inducing kinase: a key regulator in osteopontin- induced MAPK/lkappaB kinase dependent NF-kappaB-mediated promatrix metalloproteinase-9 activation. Glycoconj $J$ 2006; 23: 221-232.

11 Yokosaki Y, Matsuura N, Sasaki T, Murakami I, Schneider H, Higashiyama $\mathrm{S}$ et al. The integrin alpha(9)beta(1) binds to a novel recognition sequence (SVVYGLR) in the thrombin-cleaved amino-terminal fragment of osteopontin. J Biol Chem 1999; 274: 36328-36334.

12 Egusa H, Kaneda Y, Akashi Y, Hamada Y, Matsumoto T, Saeki M et al. Enhanced bone regeneration via multimodal actions of synthetic peptide SVVYGLR on osteoprogenitors and osteoclasts. Biomaterials 2009; 30: 4676-4686.

13 Standal T, Borset M, Sundan A. Role of osteopontin in adhesion, migration, cell survival and bone remodeling. Exp Oncol 2004; 26: 179-184.

14 Weber GF, Ashkar S, Glimcher MJ, Cantor H. Receptor--ligand interaction between CD44 and osteopontin (Eta-1). Science 1996; 271: 509-512.

15 He B, Mirza M, Weber GF. An osteopontin splice variant induces anchorage independence in human breast cancer cells. Oncogene 2006; 25: 2192-2202.

16 Jin YC, Lee H, Kim SW, Kim ID, Lee HK, Lee Y et al. Intranasal delivery of RGD motif-containing osteopontin icosamer confers neuroprotection in the postischemic brain via $\alpha \vee \beta 3$ integrin binding. Mol Neurobiol 2016; 53 : 5652-5663.

17 Kim SW, Lee HK, Kim ID, Lee H, Luo L, Park JY et al. Robust neuroprotective effects of 2-((2-oxopropanoyl)oxy)-4-(trifluoromethyl)benzoic acid (OPTBA), a HTB/pyruvate ester, in the postischemic rat brain. Sci Rep 2016; 6: 31843.

18 Dai J, Peng L, Fan K, Wang H, Wei R, Ji G et al. Osteopontin induces angiogenesis through activation of PI3K/AKT and ERK $1 / 2$ in endothelial cells. Oncogene 2009; 28: 3412-3422.

19 Ortiz-Martínez F, Sanmartín E, Pomares-Navarro E, Pérez-Balaguer A, Andrés L, Sánchez-Payá $\mathrm{J}$ et al. Osteopontin regulates VEGFA and ICAM-1 mRNA expression in breast carcinoma. Am J Clin Pathol 2015; 143: 812-822.
20 Wu XL, Lin KJ, Bai AP, Wang WX, Meng XK, Su XL et al. Osteopontin knockdown suppresses the growth and angiogenesis of colon cancer cells. World J Gastroenterol 2014; 20: 10440-10448.

21 Takahashi K, Takahashi F, Tanabe KK, Takahashi H, Fukuchi Y. The carboxyl-terminal fragment of osteopontin suppresses arginine-glycineasparatic acid-dependent cell adhesion. Biochem Mol Biol Int 1998; 46: 1081-1092.

22 Senger DR, Perruzzi CA, Papadopoulos-Sergiou A, Van de Water L. Adhesive properties of osteopontin: regulation by a naturally occurring thrombin-cleavage in close proximity to the GRGDS cell-binding domain. Mol Biol Cell 1994; 5: 565-574.

23 Hamada Y, Nokihara K, Okazaki M, Fujitani W, Matsumoto T, Matsuo M et al. Angiogenic activity of osteopontin-derived peptide SVVYGLR. Biochem Biophys Res Commun 2003; 310: 153-157.

24 Hamada Y, Yuki K, Okazaki M, Fujitani W, Matsumoto T, Hashida MK et al. Osteopontin-derived peptide SVVYGLR induces angiogenesis in vivo. Dent Mater J 2004; 23: 650-655.

25 Uchinaka A, Kawaguchi N, Hamada Y, Mori S, Miyagawa S, Saito A et al. Transplantation of myoblast sheets that secrete the novel peptide SVVYGLR improves cardiac function in failing hearts. Cardiovasc Res 2013; 99: 102-110.

26 Uchinaka A, Hamada Y, Mori S, Miyagawa S, Saito A, Sawa Y et al. SVVYGLR motif of the thrombin-cleaved $\mathrm{N}$-terminal osteopontin fragment enhances the synthesis of collagen type III in myocardial fibrosis. Mol Cell Biochem 2015; 408: 191-203.

27 Barry ST, Ludbrook SB, Murrison E, Horgan CM. Analysis of the alpha4beta1 integrin-osteopontin interaction. Exp Cell Res 2000; 258: 342-351.

28 Green PM, Ludbrook SB, Miller DD, Horgan CM, Barry ST. Structural elements of the osteopontin SVVYGLR motif important for the interaction with alpha(4) integrins. FEBS Lett 2001; 503: 75-79.

29 Niu X, Zhao L, Li X, Xue Y, Wang B, Lv Z et al. B3-Adrenoreceptor stimulation protects against myocardial infarction injury via eNOS and nNOS activation. PLOS ONE 2014; 9: e98713.

30 Oyama N, Yagita Y, Kawamura M, Sugiyama Y, Terasaki Y, Omura-Matsuoka $E$ et al. Cilostazol, not aspirin, reduces ischemic brain injury via endothelial protection in spontaneously hypertensive rats. Stroke 2011; 42: 2571-2577.

31 Ellison JA, Velier JJ, Spera P, Jonak ZL, Wang X, Barone FC et al. Osteopontin and its integrin receptor alpha(v)beta3 are upregulated during formation of the glial scar after focal stroke. Stroke 1998; 29: 1698-1706; discussion 1707.

32 Ophascharoensuk V, Giachelli CM, Gordon K, Hughes J, Pichler R, Brown P et al. Obstructive uropathy in the mouse: role of osteopontin in interstitial fibrosis and apoptosis. Kidney Int 1999; 56: 571-580.

33 Shin YJ, Kim HL, Choi JS, Choi JY, Cha JH, Lee MY. Osteopontin: correlation with phagocytosis by brain macrophages in a rat model of stroke. Glia 2011; 59: 413-423.

34 Shin YJ, Kim HL, Park JM, Cho JM, Kim CY, Choi KJ et al. Overlapping distribution of osteopontin and calcium in the ischemic core of rat brain after transient focal ischemia. J Neurotrauma 2012; 29: 1530-1538.

35 Yan YP, Lang BT, Vemuganti R, Dempsey RJ. Osteopontin is a mediator of the lateral migration of neuroblasts from the subventricular zone after focal cerebral ischemia. Neurochem Int 2009; 55: 826-832.

This work is licensed under a Creative Commons Attribution 4.0 International License. The images or other third party material in this article are included in the article's Creative Commons license, unless indicated otherwise in the credit line; if the material is not included under the Creative Commons license, users will need to obtain permission from the license holder to reproduce the material. To view a copy of this license, visit http://creativecommons. org/licenses/by/4.0/

(C) The Author(s) 2018

Supplementary Information accompanies the paper on Experimental \& Molecular Medicine website (http://www.nature.com/emm) 